\section{AB1011 CLINICAL TRIAL OF INTRAVENOUS INFUSION OF FUCOSYLATED BONE MARROW MESENCHYMAL STEM CELLS IN PATIENTS WITH OSTEOPOROSIS}

N. Lozano-Rivas ${ }^{1}$, L.F. Linares ${ }^{1}$, C. Marras-Fernandez-Cid ${ }^{1}$, A.M. GarciaHernandez $^{2}$, M.D.C. Algueró ${ }^{2}$, F. Iniesta ${ }^{2}$, D. Sanchez-Salinas ${ }^{2}$, M.D. LópezLucas $^{2}$, M. Rodriguez-Valiente ${ }^{2}$, V. Cabañas ${ }^{2}$, D. García-Bernal' ${ }^{2}$, M.D.M. Molina ${ }^{2}$, S. Lopez ${ }^{2}$, F. Ramirez-Tovar ${ }^{2}$, J.E. Ruiz Sará ${ }^{1}$, B. García ${ }^{3}$, M. Blanquer ${ }^{2}$, J. A. Olmo Fernandez-Delgado ${ }^{4}$, M. Espinosa ${ }^{5}$, J. Zamarro ${ }^{5}$, J. Becerra-Ratia ${ }^{6}$, J. L. Peris ${ }^{7}$, I. López-Exposito ${ }^{8}$, J.A. Bafalliu ${ }^{8}$, F. Ruiz-Espejo ${ }^{9}$, E. Domenech ${ }^{10}$, M. D. Morales-Cano ${ }^{10}$, P.M. Arrabal ${ }^{6}$, G. Soler ${ }^{8}$, A. Vera ${ }^{8}$, F. Guzman-Aroca ${ }^{10}$, J. M. Moraleda ${ }^{2}$, R. Sackstein ${ }^{11} .{ }^{1}$ Rheumatology Department. University Hospital Virgen Arrixaca; ${ }^{2}$ Hematopoietic Transplant and Cellular Therapy Unit, University of Murcia; ${ }^{3}$ Internal Medicine Service of the University Hospital Virgen de la Arrixaca; ${ }^{4}$ Rehabilitation Service of the University Hospital Virgen de la Arrixaca; ${ }^{5}$ Interventional Neuroradiology Service of the University Hospital Virgen de la Arrixaca, Murcia; ${ }^{6}$ BIONAND, University of Malaga, Malaga; ${ }^{7}$ Biomechanics Institute of Valencia, Valencia; ${ }^{8}$ Cytogenetics Section of Biochemistry Center and Clinical Genectic of of the University Hospital Virgen de la Arrixaca; ${ }^{9} \mathrm{Chemical}$ Analysis Service of the University Hospital Virgen de la Arrixaca; ${ }^{10}$ Radiology Service of the University Hospital Virgen de la Arrixaca, Murcia, Spain; ${ }^{11}$ Program of Excellence in Glycosciences, Harvard Medical School, Harvard University, Boston, USA

Background: Osteoporosis (OP) is a systemic bone disease characterised by decreased bone mass and deterioration of bone microarchitecture with increased brittleness and fracture risk. It associates high morbidity and mortality for patients and has a high impact on health expenditure. Bone marrow stromal mesenchymal stem cells (BM-MSC) give rise to osteoprogenitor cells and osteoblasts and influence bone homeostasis. However after their intravenous (i/v) infusion their osteotropism is limited. Our group has demonstrated that the exofucosylation of the CD44 membrane antigen in MSC improves their homing to bone tissue and that the infusion of these cells is safe in a murine model.

Objectives: To evaluate the safety of $i / v$ infusion of fucosylated BM-MSC in patients with OP, and secondarily assess their ability to improve the course of the disease

Methods: 10 women between 50 and 75 years old diagnosed with osteoporosis with a low impact fracture will be included and treated $\mathrm{i} / \mathrm{v}$ with autologous fucosylated BM-MSC. The first 4 patients were treated with a dose of $2 \times 10^{6} \mathrm{cells} / \mathrm{kg}$ body weight and the other 6 with $5 \times 10^{6}$ cells $/ \mathrm{kg}$ body weight. A 24 month followup will be conducted to evaluate the rate of severe and non-serious adverse events and secondary endpoints (decreased fracture rate, pain scores, functional status and quality of life, biochemical indexes of bone metabolism, quantitative computed tomography for morphometric and mechanical analysis of bone quality, densitometry, and histomorphometry

Results: Seven patients have been recruited to date. Two left the study for lack of cell proliferation and appearance of a complex form in karyotype during the cell culture, respectively. The first 4 patients were successfully infused, and after a median follow-up of 3 months no related adverse effects have been observed, no new osteoporotic fractures have appeared, and the analogue pain scale score (EVA) shows a tendency to decrease of pain in 3 of the 4 patients.

Conclusions: Our preliminary data indicate that clinical and GMP-grade production of BM-MSC is feasible. We have not observed any short-term adverse effects associated with treatment in infused patients.

Disclosure of Interest: None declared

DOI: 10.1136/annrheumdis-2018-eular.4728

\section{AB1012 BONE MASS CHANGES CORRELATE WITH DISEASE ACTIVITY IN NON-ARTHROPATHIC PSORIATIC PATIENTS}

S. Paolino, E. Gotelli, G. Pacini, E. Alessandri, A. Casabella, M. Ghio, F. Cattelan, F. Goegan, M. Patané, M. Cutolo. Department of Internal Medicine, Research Laboratory and Academic Division of Clinical Rheumatology, Department of Internal Medicine, University of Genova, Genoa, Italy, Genoa, Italy

Background: Psoriasis is a chronic inflammatory skin disease, and as all inflammatory processes, it could induce osteoclast activation ${ }^{1}$ ending up with osteoporosis. It is well known that a recognised contributing factor to bone strength, independent from bone mass, is micro-architectural damage; therefore, evaluation of microarchitecture seems to be necessary to get adequate information about bone quality health. Trabecular bone score (TBS) is a new tool to assess bone quality. ${ }^{2}$ To date, studies on TBS assessment in psoriasis are lacking, even if it was suggested to be correlated with inflammation and metabolic parameters. ${ }^{3}$ Objectives: The aim of the study was to investigate bone quality and bone quantity in psoriatic patients without arthropathic involvement and its relationship with dermal disease activity (PASI).

Methods: Thirty-five psoriatic patients and thirty-four healthy controls were enrolled. We first assessed Body Mass Index (BMI), Bone Mineral Density (BMD) and Trabecular Bone Score (TBS) in both patients and healthy control. The Bone Mineral Density (BMD) $(\mathrm{g} / \mathrm{cm} 2)$ was analysed by dual-energy X-ray absorptiometry scan (Lunar Prodigy) and, using same machine, anteroposterior spine analysis was performed to evaluate TBS for L1-L4 using TBS insight Medimaps software. TBS $>1.350$ units was considered normal. Psoriatic patients were also divided in two subgroups according to skin lesion presentation (plaque psoriasis and guttate psoriasis) and dermal disease activity was measured by Psoriasis Area and Severity Index (PASI).

Results: In the psoriatic cohort (mean age $62.8 \pm 12.6$ years, BMI $27.8 \pm 3.4 \mathrm{~g} / \mathrm{m}^{2}$ disease duration $18.6 \pm 11.9$ years, PASI $8.3 \pm 7.6$ ) $36 \%$ of patients showed low bone mass, particularly, $33.3 \%$ showed osteopenia and $2.7 \%$ osteoporosis. There was not significantly statistical difference between mean BMD and TBS in psoriatic patients when compared to the healthy control group. However, in psoriatic patients a negative correlation between neck-BMD and PASI $(p=0.003)$ was detected independently of sex and age. Conversely, TBS was not statistically correlated with PASI or with BMI. It was interesting to verify that the plaque psoriasis group showed a positive correlation between PASI and age $(p=0.01)$. Conversely, PASI was negatively correlated with lumbar spine $T$-score $(p=0.01)$ and both lumbar spine and femoral neck BMD ( $p=0.04$ and $p=0.02$ respectively).

Conclusions: This pilot study suggests that in psoriasis, particularly in the plaque subgroup, the severity of skin involvement might correlate with bone mass but no with bone quality (TBS). Despite previous evidences, we can't assess any correlation between TBS and metabolic parameters. Furthers studies with larger cohorts are needed on this topic.

\section{REFERENCES:}

[1] Attia EA, et al., J Dermatol 2011; 50:30-35.

[2] Silva Bc, et al., J Bone Miner Res 2014;29:518-30.

[3] Hernandéz JL, et al., J Osteoporosis, 2016; 5747852

Disclosure of Interest: None declared

DOI: 10.1136/annrheumdis-2018-eular.6625

\section{AB1013 PREDICTION OF BONE MINERAL DENSITY CHANGES IN PATIENTS WITH RHEUMATOID ARTHRITIS}

T. Tomizawa' ${ }^{1}$, H. Ito ${ }^{1}$, M. Furu' ${ }^{1}$, M. Hashimoto ${ }^{2}$, M. Tanaka ${ }^{2}$, T. Mimori ${ }^{2}$, S. Matsuda ${ }^{1}{ }^{1}$ Orthopedic Surgery, ${ }^{2}$ Rheumatology and Clinical Immunology, Kyoto University Graduate School of Medicine, Kyoto-City, Japan

Background: Osteoporosis and its related fractures is one of the most dominant, troublesome complications in rheumatoid arthritis (RA). Newly-introduced drugs such as methotrexate and biological and targeted synthetic disease modifying anti-rheumatic drugs have decreased disease activity drastically, but the improvement of osteoporosis remains to be investigated.

Objectives: To find useful factors for bone mineral density (BMD) management of RA patients under the current treatment.

Methods: We consecutively recruited 370 RA patients treated at Kyoto University Hospital in 2012. We prospectively collected the BMD values of the lumbar spine and the distal forearm measured by dual-energy X-ray absorptiometry (DXA), blood sampling test, urinalysis including bone metabolic biomarkers and clinical parameters of the RA patients in 2012 and 2014. Multivariate regression analysis was performed after adjustment by age, sex, body mass index (BMI), steroid use, anti-osteoporosis medication. We set the annualised BMD change as an outcome variable and allotted the other parameters as explanatory variables by a stepwise procedure.

Results: The average values (minimum-maximum value) of age and BMI were $63.3(32-85)$ years and $22.1(12.3-30.0)$, respectively. Female patients and steroid users accounted for $91.1 \%$, and $41.0 \%$, respectively. Coincidentally, antiosteoporosis drug-user also reached $41.0 \%$. User of biological accounted for $30.8 \%$. The averages of disease activity score (DAS) 28-erythrocyte sedimentation rate, Health Assessment Questionnaire was $2.6(0.1-5.9)$ and $0.8(0-2.9)$, respectively. The average of total Sharp score was $122.6(0-443)$. Laboratory data showed serum tartrate-resistant acid phosphatase (TRACP) $-5 b$, serum homocysteine, serum undercarboxylated osteocalcin, bone specific alkaline phosphatase, and urinary pentosidine were $320.0(68-877) \mathrm{mU} / \mathrm{dl}, 9.7(3.2-$ 28) $\mathrm{nmol} / \mathrm{ml}, 4.8(0-23) \mathrm{ng} / \mathrm{mL}, 15.6$ (5.8-43.6) $\mu \mathrm{g} / \mathrm{L}$, and $50.0(11.5-561) \mathrm{pg} / \mathrm{ml}$, respectively. Next, we describe by the result of multiple regression analysis. The levels of serum homocysteine $(\beta=-0.19 ; 95 \% \mathrm{Cl}$ : 0.24 to $1.75 ; p=0.01)$ and antiosteoporosis drug $(\beta=-0.19 ; 95 \% \mathrm{Cl} ;-0.26$ to $-0.04 ; p=0.009)$ were consistently significant predictive variables of annualised $B M D$ change of the lumbar-spine. On the other hand, serum TRACP-5b $(\beta=-0.28 ; 95 \% \mathrm{Cl} ;-0.005$ to -0.001 ; $\mathrm{p}=0.002$ ) was significant predictive one for the distal forearm.

Conclusions: Anti-osteoporosis medication may be particularly important for lumbar spine BMD for RA patients, regardless of steroid-use. Specific biomarkers would be useful such as homocysteine as lumbar spine BMD and TRACP-5b as 